\title{
Fibrilação atrial e flutter após operação de revascularização do miocárdio: fatores de risco e resultados
}

\author{
Marco Aurélio Vilela Borges LIMA*, Marcelo Luiz Peixoto SOBRAL*, \\ Claúdio MENDES SOBRINHO*, Gilmar Geraldo SANTOS*, Noedir A. G. STOLF*
}

RBCCV 44205-553

Lima M A V B, Sobral M L P, Mendes Sobrinho C, Santos G G, Stolf N A G - Fibrilação atrial e flutter após operação de revascularização do miocárdio: fatores de risco e resultados. Rev Bras Cir Cardiovasc 2001; 16(3): 244-50.

RESUMO: Objetivo: Determinar a incidência de fibrilação atrial e flutter (FAF) no pós-operatório de revascularização do miocárdio (RM), bem como os fatores preditivos e a influência destas arritmias sobre o período de internação hospitalar.

Casuística e Métodos: Foram analisados 275 pacientes submetidos à operação de revascularização do miocárdio isolada ou associada à correção de aneurisma de ventrículo esquerdo. A idade variou de 26 a 83 anos, com média de idade de $58,7 \pm 9,5$ anos. Cento e noventa e seis pacientes $(71,3 \%)$ eram do sexo masculino.

Resultados: A incidência total de fibrilação atrial e flutter pós-operatórios foi $16,4 \%$ com pico de incidência ocorrendo no segundo e terceiro dia de pós-operatório. Idade avançada, sexo masculino e história de fibrilação atrial ou flutter no pré-operatório foram identificados como fatores preditivos independentes de fibrilação atrial ou flutter no pós-operatório. Os pacientes que apresentaram FA ou flutter no pósoperatório em média 36 horas a mais na Unidade de Terapia Intensiva (UTI) e 4,8 dias a mais hospitalizados.

Conclusão: A FA e flutter são arritmias comuns no pós-operatório de revascularização do miocárdio, tendo efeito significativo sobre os tempos de permanência na UTI e de internação hospitalar.

DESCRITORES: Fibrilação atrial, epidemiologia. Flutter atrial, epidemiologia. Revascularização miocárdica, efeitos adversos. Fibulação atrial, fatores de risco. Flulter atrial, fatores de risco. Revascularização miocárdica, fatores de risco.

\section{INTRODUÇÃO}

Fibrilação atrial e flutter (FAF) são as arritmias sustentadas mais comuns após revascularização do miocárdio (RM), tendo sido reconhecidas como a principal causa de morbidade após este tipo de operação (1). Embora as conseqüências destas arritmias não sejam ameaçadoras para a vida, elas constituem um problema clínico muito importante, uma vez que a conversão para o ritmo sinusal é freqüentemente mandatória (2). FAF após RM pode resultar em hipotensão e sintomas de insuficiência cardíaca, complicações embólicas (incidência aumentada de acidente vascular cerebral no pós-operatório), arritmias ventriculares e aumento do período de internação hospitalar ${ }^{(3,4)}$. Os fatores respon-

Trabalho realizado no Serviço do Prof. Noedir A. G. Stolf, Hospital da Beneficência Portuguesa, São Paulo, SP, Brasil. Recebido para publicação em abril de 2000.

* Do Serviço do Prof. Noedir A. G. Stolf, Hospital da Beneficência Portuguesa.

Endereço para correspondência: Marco Aurélio Vilela Borges Lima. Rua Oscar Freire, 1799, apto. 201, Pinheiros. São Paulo, SP, Brasil. CEP 05409-011. Tel.: (11) 3069-5318. e-mail: stolf @ incor.usp.br 
Lima M A V B, Sobral M L P, Mendes Sobrinho C, Santos G G, Stolf N A G - Fibrilação atrial e flutter após operação de revascularização do miocárdio: fatores de risco e resultados. Rev Bras Cir Cardiovasc 2001; 16(3): 244-50.

sáveis pela alta incidência de FAF após operação cardíaca e após RM em particular, permanecem não esclarecidos. O que é certo é que a incidência de FAF após RM, de longe excede a sua prevalência reportada na população geral e em pacientes com doença coronária aterosclerótica (1). Várias investigações, buscando determinar quais pacientes são mais suscetíveis ao desenvolvimento de FAF após $\mathrm{RM}$, têm colhido resultados conflitantes. Apenas idade avançada tem, consistentemente, sido associada com aumento no risco de FAF (3). O objetivo deste estudo foi definir a incidência e os fatores preditivos de FAF no pós-operatório, em pacientes que se submeteram a operação de RM, bem como determinar os seus efeitos sobre a morbidade, mortalidade e tempos de permanência em Unidade de Terapia Intensiva e de internação hospitalar.

\section{CASUÍSTICA E MÉTODOS}

Foram estudados 275 pacientes submetidos à operação de RM isolada (270 pacientes) ou associada à correção de aneurisma de ventrículo esquerdo (5 pacientes). Foram excluídos os pacientes que se submeteram à RM junto com tratamento cirúrgico de valvopatias e aortopatias. As reoperações representaram 1,5\% (4 pacientes) do total de pacientes estudados. A idade variou de 26 a 83 anos, com média de 58,7 $\pm 9,5$ anos. Cento e noventa e seis $(71,3 \%)$ pacientes eram do sexo masculino e $79(28,7 \%)$ pacientes do sexo feminino. Duzentos e quarenta e quatro $(88,7 \%)$ pacientes eram da raça branca, e os demais se distribuindo entre as raças amarela, negra e parda. Foi avaliada a relação de FAF pós-operatórios com parâmetros pré, intra e pós-operatórios.

As variáveis pré-operatórias estudadas incluíram idade, sexo, raça, presença de angina estável ou instável, história de insuficiência cardíaca congestiva definida pela classificação da New York Heart Association (NYHA), operação prévia de revascularização, história de tabagismo, hipertensão arterial sistêmica, diabetes, FAF (constatado através de relatório médico e/ou eletrocardiograma), infarto do miocárdio, história de doença renal, história de doença pulmonar, função do ventrículo esquerdo (avaliada através de laudo da ventriculografia, Pd2 de VE e fração de ejeção), extensão da doença coronária (uni, bi, triarterial, lesão de tronco coronário esquerdo) e medicamentos em uso (antiarrítmicos, bloqueadores de canais de $\mathrm{Ca}^{++}$, betabloqueadores, digoxina e diuréticos).

Os dados intra-operatórios coletados incluíram a duração da operação, tempo de circulação extracorpórea (CEC), tempo de pinçamento aórtico, hipotermia (a mais baixa temperatura nasofaríngea atingida com o resfriamento do paciente durante a CEC), necessidade de marcapasso atrial e/ou ventricular, necessidade de assistência circulatória mecânica no desmame do paciente da CEC (balão intra-aórtico, Bio Pump) e enxertos usados (veia safena, artéria radial esquerda, artéria torácica interna esquerda, artéria torácica interna direita, artéria gastroepiplóica direita).

Finalmente, foram pontos de estudo pós-operatório o tempo de permanência na UTI após a RM, necessidade de retorno do paciente para UTI após alta para enfermaria, tempo de internação hospitalar após a operação, mortalidade e ocorrência de FAF após RM.

Análises univariadas dos resultados foram feitas através do teste do $X^{2}$ para variáveis categóricas e teste $\mathrm{t}$ de student para variáveis contínuas.

\section{RESULTADOS}

FAF ocorreu após a RM em $45(16,4 \%)$ pacientes. Embora estas arritmias se desenvolveram no pós-operatório imediato e no primeiro dia de pósoperatório (1으 $\mathrm{PO}$ em $15(33,4 \%)$ pacientes, o pico de incidência $(48,9 \%)$ ocorreu no $2^{\circ}$ e $3^{\circ} \mathrm{PO}$. Em $2(4,4 \%)$ pacientes ocorreu FAF 6 dias após a operação (Gráfico 1). A média de idade de pacientes com FAF foi 63,8 comparado com 57,7 anos para pacientes que se mantiveram em ritmo sinusal $(p<0,0001$ e IC 95\% = 3,140 a 9,046). A distribuição da idade para todos os pacientes e apenas para aqueles com FAF está demonstrado nos Gráficos 2 e 3.

Os pacientes que desenvolveram FAF no pósoperatório, não apenas eram mais velhos como também, em sua maioria, pertenciam ao sexo masculino $(86,7 \%)$. Apenas $1(2,2 \%)$ paciente do grupo de FAF apresentava fibrilação atrial prévia, porém em nossa análise este número foi significativo estatisticamente, indicando maior possibilidade de manutenção de FAF após RM naqueles pacientes com fibrilação atrial ou flutter antes da cirurgia $(p=0,0235$ e IC $95 \%=0,9786$ a 1,069).

Não houve associações estatisticamente significantes entre FAF pós-operatório e história de angina instável, classe funcional da NYHA no préoperatório, operação prévia de RM, tabagismo, hipertensão arterial sistêmica, diabetes e história de infarto do miocárdio, doença renal ou doença pulmonar.

Também não se constatou associações entre FAF pós-operatório e função ventricular ou extensão da doença coronária. 
GRÁFICO 1

TEMPO DE ÍNICIO DE FAF

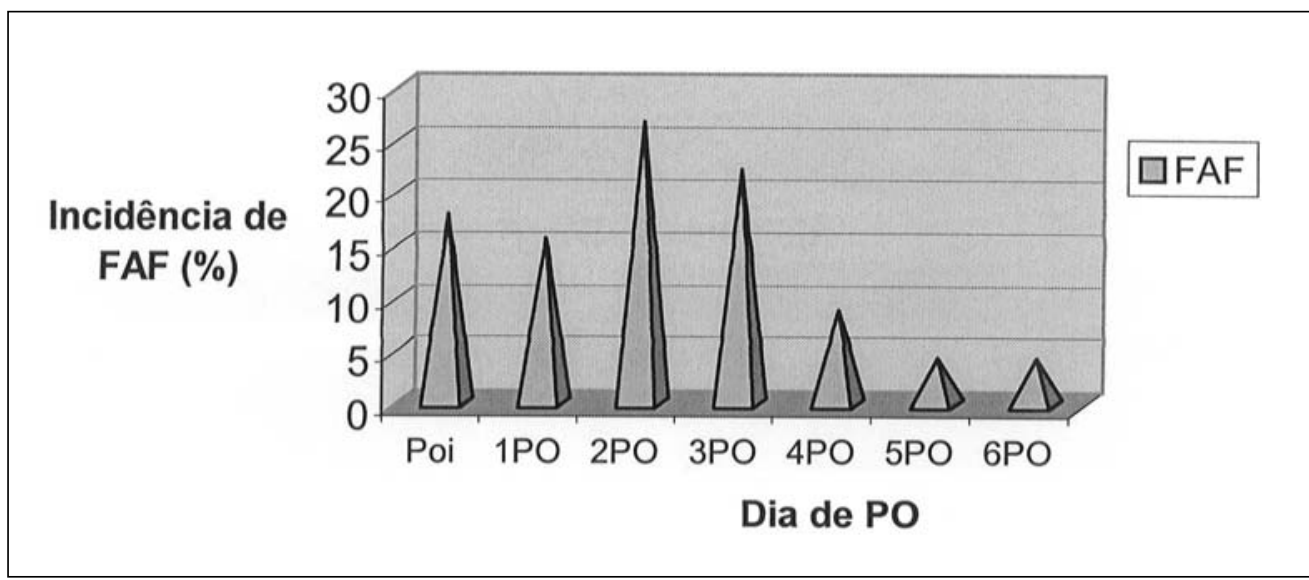

GRÁFICO 2

DISTRIBUIÇÃO DE IDADE PARA A POPULAÇÃO TOTAL

DE PACIENTES $(\mathrm{N}=275)$

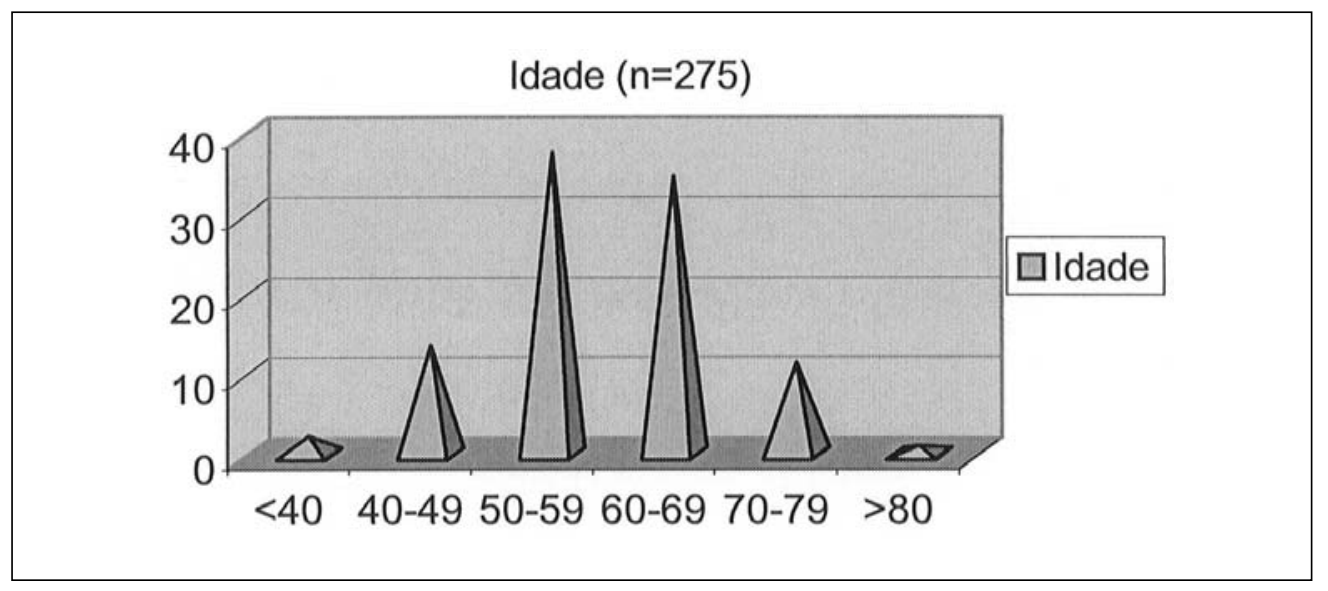

GRÁFICO 3

DISTRIBUIÇÃO DE IDADE PARA A POPULAÇÃO DE

PACIENTES COM FAF $(\mathrm{N}=45)$

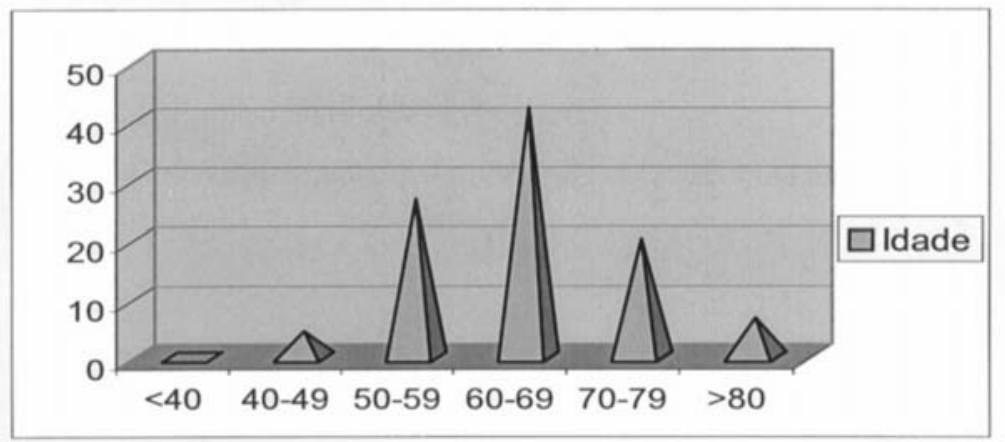


Lima M A V B, Sobral M L P, Mendes Sobrinho C, Santos G G, Stolf N A G - Fibrilação atrial e flutter após operação de revascularização do miocárdio: fatores de risco e resultados. Rev Bras Cir Cardiovasc 2001; 16(3): 244-50.

TABELA 1

\begin{tabular}{|c|c|c|c|}
\hline \multicolumn{4}{|c|}{ CARACTERÍSTICAS DOS PACIENTES NO PRÉ-OPERATÓRIO } \\
\hline CARACTERÍSTICA & $\begin{array}{c}\text { POPULAÇÃO DE } \\
\text { PACIENTES QUE } \\
\text { MANTIVERAM RITMO } \\
\text { SINUSAL NO PO } \\
(\mathrm{N}=230)\end{array}$ & $\begin{array}{l}\text { POPULAÇÃO DOS } \\
\text { PACIENTES QUE } \\
\text { DESENVOLVERAM } \\
\text { FAF NO PO } \\
(\mathrm{N}=45)\end{array}$ & P UNIVARIADO \\
\hline Idade média (anos) & 57,8 & 63,9 & $<0,0001$ \\
\hline Sexo masculino & $157(68,3 \%)$ & $39(86,7 \%)$ & 0,0126 \\
\hline Angina instável & $24(10,4 \%)$ & $5(11,1 \%)$ & 0,2 \\
\hline \multicolumn{4}{|l|}{ CF (NYHA) } \\
\hline I & $31(13,5 \%)$ & $3(6,7 \%)$ & 0,5 \\
\hline II & $137(59,6 \%)$ & $27(60,0 \%)$ & 0,5 \\
\hline III & $30(13,0 \%)$ & $8(z 17,8 \%)$ & 0,5 \\
\hline IV & $32(13,9 \%)$ & $7(15,6 \%)$ & 0,5 \\
\hline RM prévia & $3(1,3 \%)$ & $1(2,2 \%)$ & 0,6 \\
\hline Tabagismo & $142(61,7 \%)$ & $32(71,1 \%)$ & 0,2 \\
\hline HAS & $172(74,8 \%)$ & $35(77,8 \%)$ & 0,6 \\
\hline Diabete & $76(33,0 \%)$ & $10(22,2 \%)$ & 0,1 \\
\hline IAM & $125(54,3 \%)$ & $21(46,7 \%)$ & 0,3 \\
\hline Doença renal & $8(3,5 \%)$ & $1(2,2 \%)$ & 0,6 \\
\hline Doença pulmonar & $12(5,2 \%)$ & $5(11,1 \%)$ & 0,1 \\
\hline \multicolumn{4}{|l|}{ Função do VE } \\
\hline Normal & $112(48,7 \%)$ & $19(42,2 \%)$ & 0,7 \\
\hline Disfunção leve & $85(37,0 \%)$ & $18(40,0 \%)$ & 0,7 \\
\hline Disfunção moderada & $26(11,3 \%)$ & $7(15,6 \%)$ & 0,7 \\
\hline Disfunção grave & $7(3,0 \%)$ & $1(2,2 \%)$ & 0,7 \\
\hline \multicolumn{4}{|l|}{$\begin{array}{l}\text { Extensão da doença } \\
\text { coronariana }\end{array}$} \\
\hline Uniarterial & $23(10,0 \%)$ & $3(6,7 \%)$ & 0,7 \\
\hline Biarterial & $52(22,6 \%)$ & $12(26,6 \%)$ & 0,7 \\
\hline Triarterial & $144(62,6 \%)$ & $27(60,0 \%)$ & 0,7 \\
\hline Lesão de TCE & $11(4,8 \%)$ & $3(6,7 \%)$ & 0,7 \\
\hline
\end{tabular}

O uso pré-operatório de antiarrítmicos, bloqueadores de canais $\mathrm{Ca}{ }^{++}$, betabloqueadores e diuréticos não diferiu significativamente entre os pacientes que se mantiveram em ritmo sinusal e aqueles que desenvolveram FAF no pós-operatório.

A análise das variáveis intra-operatórias mostrou que duração da operação, tempo de CEC, tempo de pinçamento aórtico, necessidade de marcapasso atrial e/ou ventricular e necessidade de assistência circulatória mecânica, foram similares para o grupo de pacientes que se manteve com ritmo sinusal e para aquele que desenvolveu FAF no pós-operatório.

Os pacientes com FAF permaneceram uma média de 36 horas a mais internados na UTI e retornaram com maior freqüência para esta unidade durante a evolução pós-operatória. Após a operação, os pacientes com FAF permaneceram no hospital em média por 4,8 dias a mais do que aqueles sem FAF.

\section{COMENTÁRIOS}

Apesar dos avanços em cirurgia cardíaca de modo geral, e RM em particular, não se conseguiu diminuição na incidência de FAF no pós-operatório. Melhoramentos nas técnicas cirúrgicas, de proteção miocárdica, CEC e cuidados dos pacientes críticos na sala de operação e na UTI, têm resultado em consideráveis mudanças no perfil de risco dos pacientes revascularizados. Juntamente com a rápida 
Lima M A V B, Sobral M L P, Mendes Sobrinho C, Santos G G, Stolf N A G - Fibrilação atrial e flutter após operação de revascularização do miocárdio: fatores de risco e resultados. Rev Bras Cir Cardiovasc 2001; 16(3): 244-50.

TABELA 2

\begin{tabular}{lccc}
\hline & VARIÁVEIS INTRA-OPERATÓRIAS & \\
\hline VARIÁVEL & $\begin{array}{c}\text { PACIENTES QUE SE } \\
\text { MANTIVERAM EM } \\
\text { RITMO SINUSAL NO } \\
\text { PO (N=230) }\end{array}$ & $\begin{array}{c}\text { PACIENTES QUE } \\
\text { DESENVOLVERAM } \\
\text { FAF NO PO } \\
\text { (N=45) }\end{array}$ & P UNIVARIADO \\
\hline Tempo médio de operação (min) & 273,8 & 275,7 & 0,8 \\
\hline Tempo médio de pinçamento de Ao (min) & 55,2 & 56,1 & 0,7 \\
Tempo médio de CEC (min) & 93,2 & 97,5 & 0,3 \\
Hipotermia moderada (28-30ㄷ) & $171(74,3 \%)$ & $35(77,7 \%)$ & 0,6 \\
Uso de marcapasso atrial e/ou ventricular & $14(6,1 \%)$ & $5(11,1 \%)$ & 0,2 \\
Assistência circulatória (BIA) & $6(2,6 \%)$ & $1(2,2 \%)$ & 0,8 \\
\hline
\end{tabular}

TABELA 3

\begin{tabular}{|c|c|c|c|}
\hline \multicolumn{4}{|c|}{ VARIÁVEIS PÓS-OPERATÓRIAS } \\
\hline VARIÁVEL & $\begin{array}{c}\text { PACIENTES QUE SE } \\
\text { MANTIVERAM EM RITMO } \\
\text { SINUSAL NO PO }(\mathrm{N}=230)\end{array}$ & $\begin{array}{c}\text { PACIENTES QUE } \\
\text { DESENVOLVERAM FAF NO } \\
\text { PO }(\mathrm{N}=45)\end{array}$ & P UNIVARIADO \\
\hline $\begin{array}{l}\text { Tempo médio } \\
\text { de permanência } \\
\text { na UTI (dias) }\end{array}$ & 2,7 & 4,2 & 0,0004 \\
\hline $\begin{array}{l}\text { Retorno } \\
\text { para UTI após } \\
\text { alta para enfermaria }\end{array}$ & $4(1,7 \%)$ & $5(11,1 \%)$ & 0,0012 \\
\hline $\begin{array}{l}\text { Tempo médio } \\
\text { de internação hospitalar } \\
\text { no pós-operatório }\end{array}$ & 8,4 & 13,2 & $<0,0001$ \\
\hline Óbito & $15(6,5 \%)$ & $4(8,9 \%)$ & 0,6 \\
\hline
\end{tabular}

evolução da tecnologia e prática dos procedimentos de revascularização percutânea, os pacientes cirúrgicos atuais são significativamente mais velhos do que eram há 10 ou 15 anos, apresentando risco aumentado para maior morbi-mortalidade. A monitorização contínua têm levado a diagnósticos mais freqüentes de fibrilação atrial (FA), que permanece a causa mais comum de morbidade após revascularização do miocárdio (1).

A incidência de FA na população em geral é de cerca de $1,7 \%$, índice que aumenta progressivamente com a idade. A incidência de FA na presença de doença coronariana aterosclerótica é de 3,6\% (de acordo com Cardiovascular Health Study Collaborative Research Group), e após a maioria dos procedimentos cirúrgicos não torácicos é relatada ser de aproximadamente $5 \%(1)$. Segundo informações publicadas até 1998 , em torno de 468.000 operações de RM eram realizadas anualmente nos Estados Unidos $(4,5)$. A fibrilação atrial desenvolve-se em cerca de 18 a $33 \%$ dos pacientes que se submetem à RM, principalmente no período de 2 a 5 dias do procedimento, com pico de incidência do $2^{\circ}$ ao $4^{\circ}$ PO $(4,6)$.
Um importante e consistente fator que contribui para o aumento na incidência de FA em anos recentes é a idade avançada dos pacientes cirúrgicos. No Brigham and Women's Hospital, a média de idade dos pacientes revascularizados aumentou em 12 anos entre 1970 e 1992 e a incidência de FA aumentou, neste mesmo período, de 5 para $33 \%$. O envelhecimento produz mudanças estruturais, tais como fibrose e dilatação atrial, que parecem ser responsáveis pelo desenvolvimento de arritmias (1).

Embora em nosso estudo não tenhamos encontrado associação entre história de HAS e FAF pósoperatório, é conhecido o poder da sobrecarga pressórica em causar hipertrofia. Inúmeras mudanças no coração hipertrófico, tal como fibrose miocárdica, podem agir como substrato para arritmias de reentrada. Estudos com biópsia demonstraram anormalidades eletrofisiológicas no coração hipertrófico, causando despolarização, velocidades de condução e propagação do impulso anormais, predispondo a arritmias reentrantes. Além disso, a fibrose miocárdica pode causar um declínio na função diastólica e complacência do ventrículo esquer- 
Lima M A V B, Sobral M L P, Mendes Sobrinho C, Santos G G, Stolf N A G - Fibrilação atrial e flutter após operação de revascularização do miocárdio: fatores de risco e resultados. Rev Bras Cir Cardiovasc 2001; 16(3): 244-50.

do (1). Sabendo-se que pacientes idosos são mais provavelmente hipertensos e que HAS e hipertrofia ventricular se mostraram fatores preditivos independentes de FA no pós-operatório ${ }^{(1,6)}$, podemos assim identificar os mecanismos pelos quais o enveIhecimento favorece ao desenvolvimento de FAF pósoperatório.

A predominância do sexo masculino dentre os pacientes que desenvolvem FAF no pós-operatório tem sido relatada em outros trabalhos, sendo referido um aumento de $41 \%$, para homens, na probabilidade de desenvolvimento de FAF no pós-operatório. Os fatores que podem responder por esta distinção de risco baseada no sexo compreendem diferenças na expressão dos canais de íon, efeitos hormonais sobre o tônus automático e diferenças na arquitetura tridimensional do miocárdio ou orientação da fibra (1).

Parece que há mecanismos indeterminados que predispõem uma larga proporção de pacientes revascularizados a desenvolver FA. Alguns destes mecanismos responsáveis incluem suspensão do uso de betabloqueadores (administração peri-operatória de betabloqueadores tem sido associada a decréscimo na incidência FAF pósoperatório), uso de CEC, proteção atrial inadequada (Cox postulou que isquemia atrial seja provavelmente o gatilho responsável pelo desenvolvimento de FAF no pós-operatório) e excesso de manipulação do átrio direito (1-3,7). Porém, muitos pacientes sujeitos a estas mesmas condições mantêm ritmo sinusal no pós-operatório. A explicação se torna difícil então. Uma teoria mais atrativa e plausível seria a preexistência de anormalidades eletrofisiológicas que seriam amplificadas durante a operação. Estas anormalidades eletrofisiológicas parecem ser responsáveis pela vulnerabilidade aumentada de certos pacientes ao desenvolvimento de FA pós-operatório. Tal vulnerabilidade é baseada no conceito de "dispersão de refratariedade", que descreve a não uniformidade de um número infinito de períodos refratários atriais locais. Assim, em um paciente não vulnerável, teríamos uma transição gradual de uma região atrial de período refratário mais curto para outra região atrial de período refratário mais longo. Já em pessoas vulneráveis, ambas as regiões estariam em íntima proximidade. É mais provável que mecanismos múltiplos sejam responsáveis pelo desenvolvimento destas arritmias (1).

FA após RM tem sido associada a um aumento significativo do período de internação hospitalar (1,3,4, 6-8). É difícil, contudo, estabelecer se FA tem uma relação de causa com hospitalização prolongada ou se esta arritmia é apenas um marcador para pacientes mais complicados. Em um estudo feito por pesquisadores da University of Washington e da Oregon Health Sciences University, observou-se que o tratamento específico de FA, sozinho, provavelmente respondia por apenas uma pequena porção das hospitalizações prolongadas associadas com nova FA no pós-operatório. Apesar deste achado, concluíram que os tempos prolongados de permanência hospitalar daqueles pacientes que desenvolveram FA e o poderoso valor preditivo de FA para explicar estas internações prolongadas, mesmo após correção para outras variáveis clínicas, sugerem que FA não é simplesmente um marcador para pacientes mais complicados, mas de uma maneira causal, contribui para manter o paciente por mais tempo no hospital (8).

Identificar e tratar pacientes com risco elevado de desenvolvimento de FAF no pós-operatório de RM, utilizando-se, para esse propósito, medidas profiláticas agressivas e também drogas que controlem rapidamente a arritmia, é desejável para minimizar as taxas de morbidez e, possivelmente, para abreviar o tempo de internação hospitalar $(1,3,4,8)$.

Em conclusão, a FAF são arritmias muito comuns após $R M$, sendo responsáveis por aumento da morbidade e dos tempos de permanência na UTI e de internação hospitalar pósoperatória. A devida consideração destes fatos certamente resultará em melhor e mais resumida evolução pós-operatória dos pacientes revascularizados. 
Lima M A V B, Sobral M L P, Mendes Sobrinho C, Santos G G, Stolf N A G - Fibrilação atrial e flutter após operação de revascularização do miocárdio: fatores de risco e resultados. Rev Bras Cir Cardiovasc 2001; 16(3): 244-50.

RBCCV 44205-553

Lima M A V B, Sobral M L P, Mendes Sobrinho C, Santos G G, Stolf N A G - Atrial fibrillation and flutter following coronary artery bypass graft surgery : risk factors and results. Rev Bras Cir Cardiovasc 2001; 16(3): 244-50.

ABSTRACT: Objective: The aim of this study was to determine the overall incidence and predictors of atrial fibrillation and flutter (AFF) following CABG, as well as the influence of these arrhythmia on the hospital length of stay.

Material and Methods: Two hundred and seventy-five patients, who had undergone coronary artery bypass graft (CABG) surgery at the Beneficência Portuguesa Hospital in São Paulo, had their data collected and analyzed. The age range was from 26 to 83 years old with mean age of 58.7 and standard deviation of 9.5 years. One hundred and ninety six patients $(71.3 \%)$ were male.

Results: The outcomes of this analysis were: the overall incidence of postoperative AFF was $16.4 \%$, with the peak rate in the second and third postoperative days. Advanced age $(p<0.0001 ; 95 \%$ confidence interval $[\mathrm{Cl}], 3,140$ to 9.046$)$; male sex $(p=0.0126$; odds ratio [OR], 3.022; relative risk $=2.380$; $95 \% \mathrm{Cl} 1.103$ to 5,135$)$ and a history of AFF $(p=0.0235$, OR $=15.54$, relative risk $=1.023,95 \%$ $\mathrm{Cl} 0.6225$ to 387.9 ) were identified as independent predictors of postoperative AFF. Those patients with postoperative AFF remained an average of 36 hours longer in the intensive care unit and 4.8 days longer in the ward when compared with patients without AFF.

Conclusions: The AFF are very common arrhythmia after CABG and have a significant effect on both intensive care unit and overall hospital length of stay.

DESCRIPTORS: Atrial fibrillation, epidemiology. Atrial flutter, epidemiology. Myocardial revascularization, adverse effects. Atrial fibrillation, risk factors. Atrial flutter, risk factors. Myocardial revascularization, risk factors.

\section{REFERÊNCIAS BIBLIOGRÁFICAS}

1 Aranki S F, Shaw D P, Adams D H et al. - Predictors of atrial fibrillation after coronary artery surgery: current trends and impact on hospital resources. Circulation 1996; 94: 390-7.

2 Frost L, Molgaard H, Christiansen E H, Hjortholm K, Paulsen $\mathrm{P} \mathrm{K}$, Thomsen $\mathrm{P} E$ - Atrial fibrillation and flutter after coronary artery bypass surgery: epidemiology, risk factors and preventive trials. Int $J$ Cardiol 1992; 36: 253-61.

3 Mathew J P, Parks R, Savino J S et al. - Atrial fibrillation following coronary artery bypass graft surgery: predictors, outcomes and resource utilization. JAMA 1996; 276: 300-6.

4 Tisdale J E, Padhi I D, Goldberg A D et al. - A randomized, double-blind comparison of intravenous diltiazem and digoxin for atrial fibrillation after coronary artery bypass surgery. Am Heart J 1998, 135(5Pt 1): 739-47.
5 Kupersmith J, Holmes-Rovner M, Hogan A, Rovner D, Gardiner J - Cost- effectiveness analysis in heart disease, part III: ischemia, congestive heart failure and arrhytmias. Prog Cardiovasc Dis 1995; 37: 307- 46.

6 Chung M K, Asher C R, Dykstra D et al. - Atrial fibrillation increases length of stay and cost after cardiac surgery in low risk patients targeted for early discharge. J Am Coll Cardiol 1996; 27: 309A. (Abstract).

7 Fuller J A, Adams G G, Buxton B - Atrial fibrillation after coronary artery bypass grafting: is it a disorder of the elderly? J Thorac Cardiovasc Surg 1989; 97: 821-5.

8 Raitt M H, Dolack L, Kino K, Hofer B, Bardy G H Atrial fibrillation is strongly associated with prolonged hospital stay after open heart surgery even after correcting for common predictors of morbidity. J Am Coll Cardiol 1995; 25: 395A. (Abstract). 